\title{
A Method for Separating Crystallograpically Similar Phases in Steels using EBSD and Machine Learning
}

\author{
J. Goulden ${ }^{1}$, K. Mehnert ${ }^{2}$, K. Thomsen ${ }^{2}$, H. Jiang ${ }^{1}$ \\ 1. Oxford Instruments NanoAnalysis, Halifax Road, High Wycombe, UK \\ 2. ST Development ApS, Samsøgade 83, Aarhus, Denmark
}

The relationship between the mechanical and physical properties of metallic materials and microstructure is well documented; for example through the Hall-Petch relationship, where strength is inversely dependent on the square root of the grain diameter [1].

In addition to grain size, another important characteristic of steels is phase fraction identification and quantification. The relative fractions of different phases will influence the behavior of the steel in different regimes. In some cases individual or secondary phases are readily identified using X-ray microanalysis, or a combination of X-ray analysis and EBSD.

However, distinguishing crystallographically similar phases in steel is a recognized challenge with EBSD, as for example with martensite and ferrite. These phases are both fcc structured and are so crystallographically and chemically similar that it is not possible to differentiate them by either EBSD or EDS.

However, martensite generally has a distorted crystal lattice with a higher density of crystalline defects or residual stresses (generated during processing). As a result the EBSD pattern generated from these phases is of a poorer quality, in terms of intensity and sharpness, than that collected from ferrite. Therefore discrimination of these phases has focused on the analysis of EBSD pattern quality parameters, including band slope (BS), band contrast (BC) and pattern quality (PQ). Each of these measures can be plotted as a grey scale map, where martensite appears darker with a lower pattern quality when compared to ferrite, an example is shown in Figure $1 \mathrm{a} b$ and $\mathrm{c}$.

Here a technique which applies these pattern quality measures to aid in identifying these phases is demonstrated. A multitude of parameters that help distinguish different phases can be used at in combination, e.g. different pattern quality values. In the case of martensite and ferrite a bimodal distribution in pattern quality results from the presence of the relatively lower strain ferrite matrix (with a higher quality measure) and typically higher strain martensite. This can then be applied to differentiate these phases shown in Figure 2.

This method requires regions on a EBSD map to be associated with specific phases. In this way the software is presented with examples of correct phase assignment, and is trained to further phase discrimination automatically. Those phases which are judged as correctly assigned by the initial EBSD analysis can be 'locked' to prevent any changes by the subsequent automatic phase discrimination. The software training may be an interactive process, so that the results of the automatic phase discrimination are viewed, and more training undertaken if required. Assigning phase IDs to unclassified map positions is performed by applying a variant of the Nearest Neighbour method [2] in a multidimensional parameter space. The use of this method to automatically quantify phase fractions is demonstrated. A description of this method with further application examples will be shown. 


\section{References:}

[1] F. J. Humphreys, Journal of Materials Science 36 (2001), p. 3833.

[2] METU Informatics Institute, Min720, Pattern Classification with Bio-Medical Applications. Part 6: Nearest and k-nearest Neighbor Classification http://ocw.metu.edu.tr/pluginfile.php/4877/ mod_resource/content/1/Min720lecturenotes_3.pdf

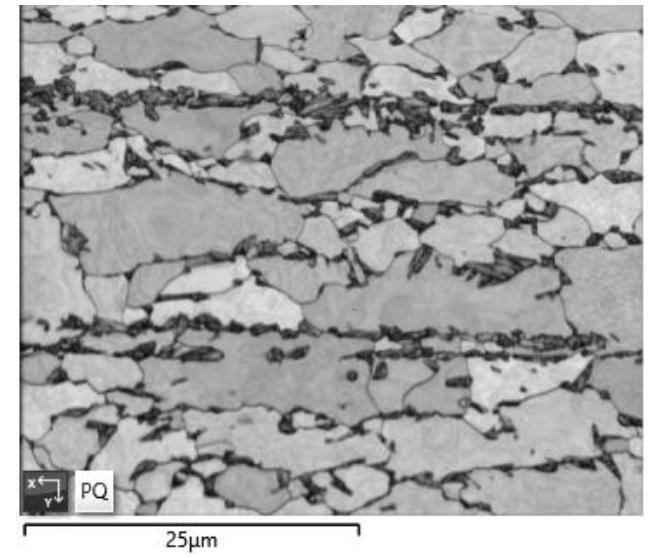

Figure 1a. Pattern Quality Map of a steel, darker regions have a lower pattern quality

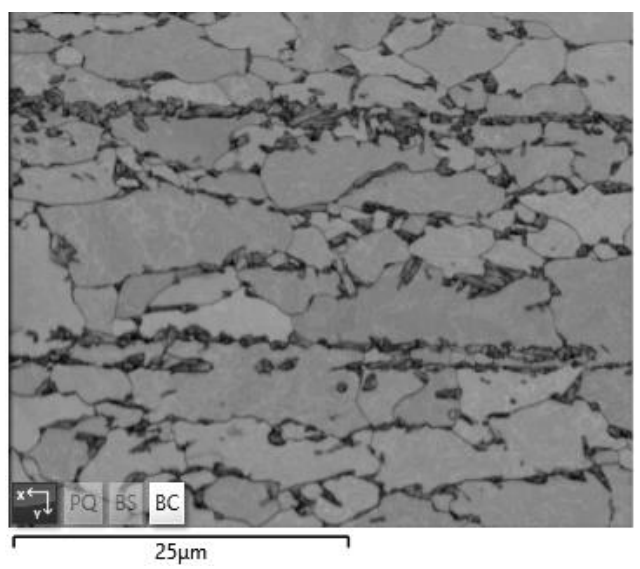

Figure 1c. Band Contrast Map of the steel

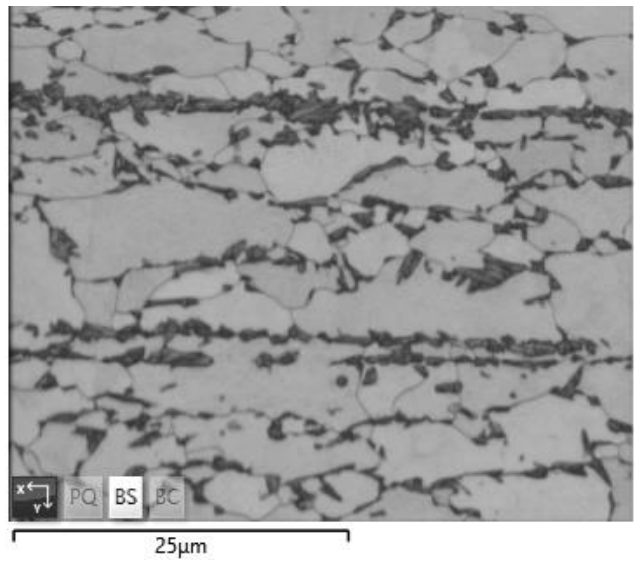

Figure 1b. Band Slope Map of the steel

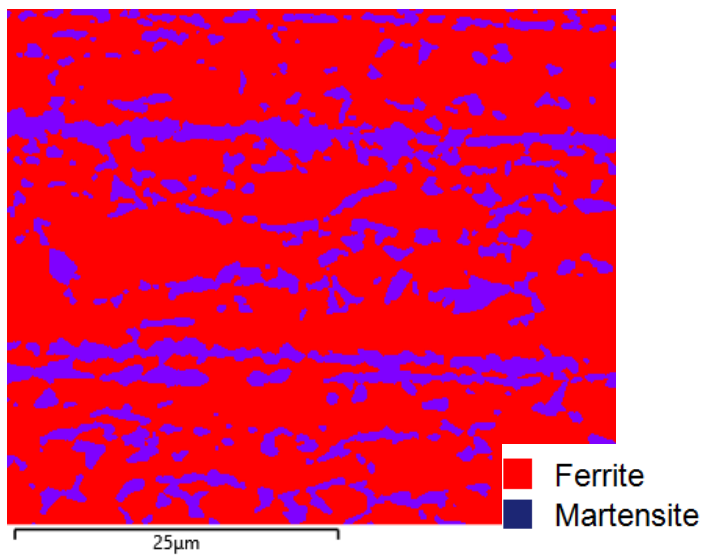

Figure 2. Ferrite and Martensite phase map 